\title{
Decisiones multisectoriales en tiempos de estado de emergencia nacional por Covid-19, Región Tacna
}

\author{
Multisectoral decisions in times of national state of emergency for Covid- \\ 19 Tacna region
}

${ }^{1}$ Oscar Alfredo Begazo Portugal

${ }^{2}$ Jehovanni Fabricio Velarde Molina
ORCID: 0000-0001-7787-1742

ORCID: 0000-0002-4382-1736

\section{RESUMEN}

El presente artículo se realiza ante un marco de Estado de Emergencia Nacional, según Decreto Supremo N ${ }^{\circ} 044$ - 2020 - PCM publicado en el Diario El Peruano (2020), para luchar contra el COVID-19. En Perú, si bien es cierto se tuvo una respuesta rápida para mitigar mayores infectados por COVID-19, los resultados no han acompañado a las soluciones que el Estado ha implementado. Sin embargo, las decisiones multisectoriales han tenido una mejor percepción en la población por la atención focalizada, mas no por los resultados de infectados en la región. Se ha llegado a las siguientes conclusiones: Las decisiones multisectoriales, en tiempos de estado de emergencia nacional, necesitan de estrategas (decisores que tomen decisiones estratégicas en beneficio de la población). Sin estos decisores con visión integral y controles, se generará un distanciamiento entre el gobierno central y los gobiernos regionales, ya que no se propiciará el vínculo necesario entre ellos. Las respuestas serán muy lentas por falta de liderazgo y comunicación. La gestión política tiene una relación directa con las estrategias nacionales, en ese contexto, nuestras autoridades político-civiles y autoridad político militar deben de trabajar de la mano y concatenadamente para salir vencedores en esta pandemia.

Palabras clave: Decisiones multisectoriales, estrategas, gestión política, interés nacional.

\begin{abstract}
This article is carried out against a framework of a National State of Emergency according to Supreme Decree No. 044 - 2020 - PCM published in the Diario el Peruano (2020) to fight against COVID-19, in the case of Peru although it is true that had a rapid response to mitigate older people infected with COVID-19 the results have not accompanied the solutions that the State has implemented, however, in multisectoral decisions they have had a better perception with the population, due to the focused attention but not the results of infected in the region. The following conclusions have been reached. Multisectoral decisions in times of a state of national emergency need strategists (decision-makers who make strategic decisions for the benefit of the population). Without these decision-makers with an integral vision and controls, a distance will be generated between the central government and the regional governments, since the necessary link between them will not be fostered. And the responses will be very slow due to lack of leadership and communication. Political management has a direct relationship with national strategies, it is in this context where our civil political authorities and military political authority must work hand in hand and concatenated to win in this pandemic.
\end{abstract}

Keywords: Political management, multisectoral decisions, strategists, national interest.

\footnotetext{
${ }^{1}$ Universidad Nacional Jorge Basadre Grohmann. Tacna, Perú. E-mail: begazop@ yahoo.com

${ }^{2}$ Universidad Nacional Jorge Basadre Grohmann. Tacna, Perú. E-mail: jvelardem@ unjbg.edu.pe 


\section{INTRODUCCIÓN}

En situaciones donde la población necesita de estrategas y líderes que puedan tomar decisiones rápidas y oportunas para poder hacer frente a la pandemia, nos encontramos con autoridades político-civiles faltos de sentido integrador, indolentes frente al sufrimiento e incertidumbre de la población que en algún momento les dio su respaldo.

En ese contexto, nos encontramos en una disyuntiva de encontrar soluciones a los problemas sanitarios por parte nuestras autoridades político-civiles regionales o esperar que el poder ejecutivo tome acción, siendo la incapacidad, inoperancia y falta de gestión política, las principales características de nuestras autoridades. Es por ello que destacamos los mejores y mayores avances respecto a las decisiones multisectoriales que el Estado pudo tomar y logró mayor impacto en la población de la región de Tacna. Fue la participación de las instituciones gubernamentales las que, de forma coordinada, hicieron funcionar lo planteado, siendo la estrategia el medio para hacer realidad las soluciones que busca el pueblo.

\section{MÉTODO}

Para realizar el presente artículo se han utilizado fuentes primarias, para enriquecer la información y orientar la investigación plasmada. Por ello, en la comunidad científica académica se lo denomina como artículo teórico. Es de tipo básico de investigación cualitativa, de diseño narrativo, ya que se contextualiza la época y lugar donde ocurrieron las experiencias y reconstruye historias individuales, los hechos, la secuencia de eventos y los resultados e identifica categorías y temas en los datos narrativos, para finalmente entretejerlos y armar una historia o narrativa general (Hernández Sampieri, 2014).

\section{DESARROLLO TEMÁTICO}

El presente artículo se realiza frente a un marco de Estado de Emergencia Nacional según Decreto Supremo No 044 - 2020 - PCM publicado en el Diario El Peruano (2020), para luchar contra el COVID-19, virus que azota a todo el mundo. Esta pandemia tuvo inicio en la República Popular China, donde existen reportes de infectados desde el mes de noviembre; sin embargo, oficialmente su primer infectado fue en diciembre en la ciudad de Wuhan. Según el Portal Web Pandemia de enfermedad por coronavirus de 2019-2020, en el caso de Perú el primer infectado fue reportado oficialmente el 5 de marzo, pero fue el 16 de marzo cuando se decreta el Estado de Emergencia Nacional, con 86 infectados.

En este contexto, buscamos responder por qué lo sucedido en este año 2020 fue el detonante para dejar en evidencia los sectores que se tuvieron en total abandono durante los últimos 10 o 15 años (sector salud, sector educación, sector comercio, sector defensa y sector del interior. Del mismo modo, la falta de una solución multisectorial que refleje una rápida respuesta por parte de las autoridades de la región Tacna y en general el comportamiento de nuestras autoridades frente a momentos de crisis donde se necesita de estrategas para tomar decisiones integradoras y soluciones oportunas. En este caso, para combatir la pandemia COVID-19, no con una mirada reactiva sino con un enfoque prospectivo integrador, donde se busque la institucionalización de cada órgano de gobierno, identificando responsabilidades y a la vez unificando esfuerzos entre las 
instituciones involucradas decisoras del futuro de la región. Asimismo se mostrará un sentido crítico de análisis buscando las principales causas y proponiendo soluciones.

Antes de entrar a fondo, se hace mención al historiador Jorge Basadre (1983), hijo predilecto de Tacna, que en su libro Historia de la República del Perú señala:

"El Estado empírico quiere decir el Estado inauténtico, frágil, corroído por impurezas y por anomalías. Es el Estado con un presidente inestable, con elecciones a veces amañadas, con un Congreso de origen discutible y poco eficaz en su acción, con democracia falsa. Estado empírico quiere decir, asimismo, que en él no abundan como debieran las gentes capaces y bien preparadas para la función que les corresponde ejercer en la administración y que no hay garantías para formar esos cuadros o para permitirles actuar. Estado empírico hasta llegar a lo increíble era el que había despilfarrado millones locamente en la época de las consignaciones y luego en la época de los grandes empréstitos para desembocar en la bancarrota. Estado empírico era el que carecía de institutos armados, medianamente organizados, de mandos competentes, oficialidad bien formada, tropa debidamente atendida, equipo moderno, servicios de administración eficientes."

Fue tan visionario Jorge Basadre, ya que esta guerra contra el COVID-19 nos encontró con un Estado no institucionalizado, desde el Ministerio de Salud, Ministerio de Defensa, Ministerio del Interior, Ministerio de Educación y Ministerio de Comercio Exterior, con distintas problemáticas y limitaciones. Sin embargo, actualmente se están haciendo esfuerzos denodados para vencer al virus y atender la cantidad de infectados.

Por otro lado, podemos observar cómo las decisiones multisectoriales pueden traer muchos beneficios a la población por su grado de compromiso e involucramiento de las instituciones en todos sus niveles (estratégico nacional, ministerial, regional, municipal y distrital). Francisco Mejía Prado, líder de la línea económica de la Mesa de Desarrollo Sostenible del Grupo Multisectorial (Grupo Multisectorial. (2020), menciona:

"A menos que los países de la región pongan la educación de calidad en el centro de sus agendas políticas, como lo hacen las naciones asiáticas, me temo que esta parte del mundo será aún más pobre y desigual que ahora."

También tenemos en la Revista Latin America \& The Caribbean (2014), donde mencionan cómo Argentina tiene un avance hacia un modelo multisectorial y define su importancia:

"La definición es interesante porque da cuenta de la autonomía relativa de cada sector, un elemento necesario para que el modelo multisectorial de gobernanza funcione como se supone debe hacerlo."

Con el mismo horizonte en el Foro Multisectorial (2018), realizado en Argentina, se manifiesta la importancia delas reuniones para la coordinación y toma de decisiones:

"Debe establecer frecuencia y formato de sus reuniones, quién establece la agenda, cómo se toman las decisiones, cómo se comparte información, cómo se 
manejan conflictos, nivel de detalle de las minutas, autoridad que toma las decisiones."

En el Estado de Colombia, a través del Ministerio de Salud (2020), se define el funcionamiento de la interacción de los sectores para lograr cumplir los objetivos nacionales, en cuanto a la salud señala que: Se fundamenta en la integración entre sectores, lo cual puede ser usado productivamente para resolver los problemas, además, permite compartir los recursos, saberes, experiencias de cada sector y posibilita la búsqueda de soluciones integrales en las políticas públicas, que buscan bienestar, desarrollo y calidad de vida de la población. En este contexto se identifica el territorio, ya sea departamento, distrito o municipio, como el espacio apto y propicio para la real operación.

Antes de la pandemia, era una cruda realidad la falta de atención al sector de educación, mientras en las noticas se podía observar las protestas sociales en diferentes países latinoamericanos que coincidían en los siguientes temas: inequidad-desigualdad, lucha contra la corrupción y mejorar con inversión el atraso educativo reflejado en las pruebas PISA. Se necesitaba con prioridad una reforma educativa en el Perú, no solo de presupuesto o de capacitación del potencial humano, sino una reforma de carácter multisectorial, donde todas las instituciones se comprometan e involucren para mejorar la educación; sin embargo, la prioridad fue desplazada por la urgencia para hacerle frente al COVID-19. En este periodo de tiempo (desde el 16 de marzo a la fecha 30 de agosto), se puede evidenciar en qué momento se toman decisiones multisectoriales en la región.

En el caso del Perú, se manejó hasta el momento tres campañas.

La primera campaña denominada “Quédate en casa", según DS No 044 - 2020 - PCM (2020), la cual consistía en permanecer en sus hogares de lunes a viernes entre las 1800 hasta las 0400 horas, y los domingo durante todo el día. Se cerraron las fronteras y restringieron transporte terrestre y los vuelos internacionales, el distanciamiento social fue obligatorio, se restringió el libre tránsito y reuniones de más de 30 personas, entre otras decisiones. Sin embargo, la respuesta de nuestras autoridades político civiles (nivel regional, municipal y distrital) fueron las menos oportunas e integradoras, no supieron responder de forma estratégica. Por ejemplo, no se implementaron las cabinas de desinfección en mercados de forma temprana, no se realizó señaléticas de distanciamiento en mercados y centros de abastos, entre otras. En el Centro de Operaciones de Emergencia Regional- COER se tomó decisiones que no tuvieron el seguimiento debido, quedando gran parte de lo acordado en letra muerta. A continuación, se pueden observar cifras sobre los infectados. 


\section{Figura 1}

Cantidad de infectados (trimestre 16/03 al 16/05)

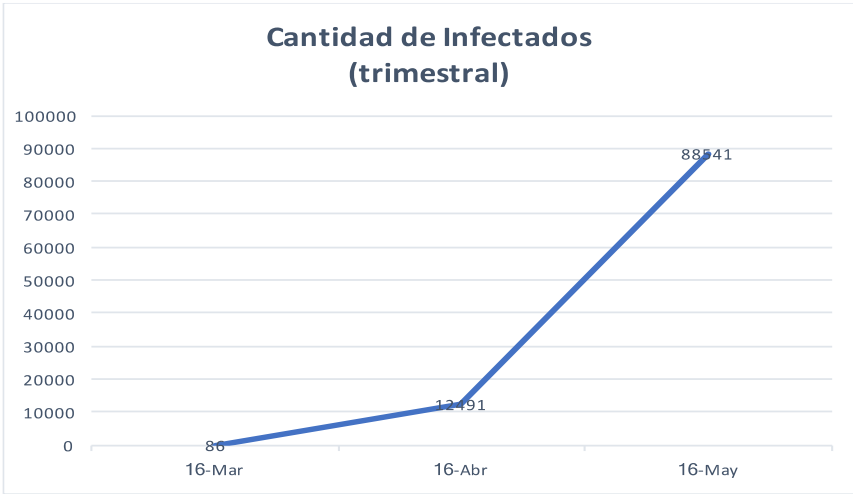

Fuente: https://www.gob.pe/coronavirus

La segunda campaña fue denomina "Usa mascarilla y Reactiva Perú", según DS No 051 - 2020 - PCM DL No 1455 (2020) y DS No 124 - 2020 - EF (2020). Esta campaña consistió en permanecer en los hogares de lunes a sábado entre las 1800 hasta las 0400 horas, y el domingo el aislamiento social fue durante todo el día. Además podían funcionar distintos sectores económicos, pero cumpliendo con protocolos de bioseguridad. Se destinaron s/. 30,000,000,000.00 para créditos y otorgamiento de garantía por parte del Estado por (90 días). Se implementó la normativa para multar a los ciudadanos que incumplan con las medidas tomadas por el Gobierno, se restringió el libre tránsito y reuniones de más de 30 personas. Ciertamente, la población se encontraba saturada y acalorada por la falta de apoyo por parte del Gobierno, a pesar de la comunicación que existía a través de las conferencias casi diarias del presidente de la Republica. Los infectados se incrementaban y la economía comenzó a desacelerar, por ende los bolsillos de la clase emergente o media ya sentía los rezagos de permanecer en aislamiento social.

\section{Figura 2}

Cantidad de infectados (trimestre 16/06 al 16/08)

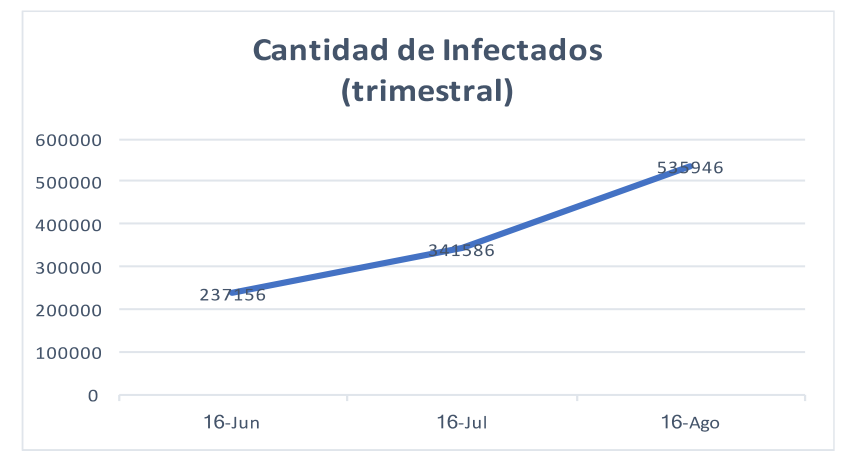

Fuente: https://www.gob.pe/coronavirus

La tercera campaña se denominó "Te cuido Perú y Plan Tayta", luego del DS No 068 2020 - PCM (2020). Esta campaña consintió en permanecer en los hogares de lunes a sábado entre las 2000 hasta las 0400 horas, y los domingos durante todo el día. Se cambió de estrategia y la atención se volvió focalizada identificando a los infectados y denominando algunas "zonas calientes" en la región. Se permitió que distintos sectores económicos puedan funcionar, cumpliendo con protocolos de bioseguridad, la normativa 
fue más rígida para multar a los ciudadanos que incumplían con las medidas tomadas por el Gobierno, se restringió el libre tránsito y las reuniones familiares.

\section{Figura 3}

Casos en el Perú (hasta el 30/08)

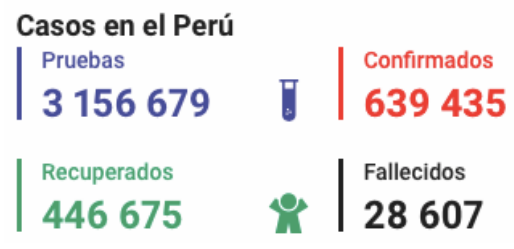

Fuente: https://www.gob.pe/coronavirus

En Tacna, se pudo percibir recién una estrategia multisectorial que pueda dar solución al problema de infectados con el plan Tayta, donde trabajaron distintas instituciones en conjunto con el Ejército Peruano, la Policía Nacional del Perú, Dirección Regional de Salud de Tacna (Diresa), INDECI y las organizaciones sociales de base, lo que permitió garantizar la efectividad de un trabajo articulado, encaminado al cumplimiento del objetivo de salvaguardar la salud de nuestros adultos mayores y población tacneña del contagio del COVID-19. Este plan se desarrolló en el centro poblado Leoncio Prado, distrito de Pocollay, distrito de Ciudad Nueva, distrito de Gregorio Albarracín y el centro poblado La Natividad.

Es necesario señalar como un aspecto positivo estas decisiones multisectoriales, ya que fueron coordinadas para el cumplimiento de objetivos y así mitigar mayores contagios.

Por otro lado, "Te cuido Perú" tiene la misión de vigilar y asistir a personas afectadas por COVID-19 que cumplen inmovilización social obligatoria las 24 horas. Este grupo de trabajo de entorno netamente multisectorial está conformado por representantes de la PCM, MININTER, MTC, MINSA, ESSALUD, CCFFAA, PNP, INDECI, RENIEC, OSIPTEL, entre otros. Entre sus responsabilidades está el diseño y puesta en funcionamiento de una plataforma digital que permite la trazabilidad de los positivos y de la población vulnerable, la detección, seguimiento médico y soporte emocional de los contagiados; una vez que se los ha identificado, el grupo está a cargo del control de su aislamiento domiciliario, la provisión de un apoyo alimentario periódico, la vigilancia de sus desplazamientos y los de su entorno, así como el tratamiento de los fallecidos. (Ejército del Perú, 2020)

Así como se implementaron acciones en beneficio para la población, también se dieron situaciones que afectaron la ascendencia y liderazgo de las autoridades político-civiles, como en el caso del gobernador de Arequipa, cuando no se tomaron decisiones que salvaguarden la salud de su región. Por ejemplo, el gobierno central tuvo que intervenir en las estrategias que se estuvieron manejando en el sistema de salud, desplazando la autoridad política civil del gobernador. Publicado en el Diario el Peruano (2020), el Decreto de Urgencia $\mathrm{N}^{\mathrm{0}}$ 086-2020 delegó estas funciones a la ministra de salud y designando a una autoridad político militar al frente del "Comando COVID", siendo parte del equipo decisor en el estado de emergencia nacional. 


\section{CONCLUSIONES}

Las decisiones multisectoriales, durante el estado de emergencia nacional, necesitan de estrategas (decisores que tomen decisiones estratégicas en beneficio de la población). Sin estos decisores con visión integral y control se generará un distanciamiento entre el gobierno central y los gobiernos regionales, ya que no se propiciará el vínculo necesario entre ellos. Asimismo, las respuestas serán muy lentas por falta de liderazgo y comunicación.

La gestión política tiene una relación directa con las estrategias nacionales, en ese contexto, nuestras autoridades político-civiles y autoridad político-militar deben de trabajar de la mano y concatenadamente para salir vencedores de esta pandemia.

\section{REFERENCIAS}

Basadre, J. (1983). Historia de la República del Perú (Sétima ed., Vol. I). Lima, Perú: Universitaria.

Diario El Peruano. (22 de Julio de 2020). www.elperuano.com.pe. Recuperado el 18 de Agosto de 2020, de El peruano: https://busquedas.elperuano.pe/normaslegales/decreto-de-urgencia-que-dictamedidas-extraordinarias-para-m-decreto-de-urgencia-n-086-2020-1872943-1/

Diario El Peruano. (2020). Recuperado el 31 de Agosto de 2020, de https://busquedas.elperuano.pe/normaslegales/decreto-supremo-que-modifica-elarticulo-3-del-decreto-supre-decreto-supremo-n-068-2020-pcm-1865516-5/

El Diario El Peruano. (2020). Recuperado el 31 de Agosto de 2020, de https://busquedas.elperuano.pe/normaslegales/prorroga-del-estado-de-emergencianacional-declarado-mediant-decreto-supremo-no-051-2020-pcm-1865180-2/

Diario El Peruano. (2020). Gobierno del Perú. Recuperado el 31 de Agosto de 2020, de www.gob.pe: https://www.gob.pe/institucion/mef/normas-legales/606067-1242020-ef

Ejercito del Perú. (8 de abril de 2020). Centros de Estudios Estratégicos del Ejército del Perú. Recuperado el 31 de Agosto de 2020, de www.ceeep.mil.pe: https://ceeep.mil.pe/2020/07/01/la-gestion-multisectorial-en-tiempos-de-crisis-yla-transformacion-del-ejercito-del-peru/

Gobierno del Perú. (2020). Gobierno del Perú. Recuperado el 31 de Agosto de 2020, de www.gob.pe/: https://www.gob.pe/coronavirus

Gobierno del Perú. (2020). Gobierno del Perú. Recuperado el 20 de 08 de 2020, de www.gob.pe/: https://www.gob.pe/institucion/pcm/normas-legales/460472-0442020-pcm

Gobierno Abierto. (21 de Mayo de 2018). Foro Multisectorial. Recuperado el 01 de Setiembre de 2020, de Foro multisectorial de la OGP - qué es, cómo se gobierna y otras cosas que no sabíamos en un bullet list de 7 puntos: http://www.agaargentina.org/2018/05/21/818/

Grupo Multisectorial. (2020). Grupo Multisectorial. Recuperado el 23 de 08 de 2020, de www.grupomultisectorial.com:

https://www.grupomultisectorial.com/noticia/america-latina-debe-repensar-sumodelo-economico-y-educativo 
Hernández, R. (2014). Metodología de la Investigación (6 ${ }^{\mathrm{a}}$ edición ed.). México D.F., México: McGraw-Hill

Latin Americana \& The Caribbean. (28 de Mayo de 2014). DIGITAL RIGHTS. Recuperado el 31 de Agosto de 2020, de www.digitalrightslac.net: https://www.digitalrightslac.net/es/la-argentina-y-el-avance-hacia-el-modelomultisectorial/

Ministerio de Salud. (2020). MINSALUD. Recuperado el 02 de Setiembre de 2020, de www.minsalud.gov.co:

https://www.minsalud.gov.co/salud/publica/epidemiologia/Paginas/comisionintersectorial-de-salud-publica.aspx 